\title{
Evaluating outcomes of mother-infant pairs using dolutegravir for HIV treatment during pregnancy
}

\author{
Clara Grayhack ${ }^{a}$, Anandi Sheth ${ }^{b}$, Olivia Kirbyc ${ }^{c}$, Jennifer Davis ${ }^{d}$, \\ Kedesha Sibliss ${ }^{\mathrm{e}}$, Hervette Nkwihoreze ${ }^{\mathrm{e}}$, Erika Aaron ${ }^{\mathrm{f}}$, Gregg Alleyneg, \\ Roberta Laguerre ${ }^{\mathrm{h}}$, Aadia Ranai, Martina Badell ${ }^{\mathrm{i}}$ \\ and Florence Momplaisir ${ }^{\mathrm{e}}$
}

\begin{abstract}
Objectives: Dolutegravir (DTG), a second-generation integrase inhibitor, is an effective treatment for HIV but its safety and efficacy are not well established in pregnancy. Here, we assess maternal and infant outcomes of mother-infant pairs using DTG-containing regimens during pregnancy.

Methods: We performed a retrospective cohort analysis of pregnant women with HIV on DTG from two urban clinics in the United States, 2015-2018. Maternal outcomes included viral suppression (viral load of $<20$ copies/ml prior to delivery), development of resistance, and tolerability to DTG. Infant outcomes included preterm delivery (birth at $<37$ weeks), small for gestational age (SGA, weight $<10$ th percentile), infant HIV status at birth, birth defect(s), and Appearance, Pulse, Grimace, Activity, Respiration (APGAR) scores. We performed a trend analysis to assess DTG use over time.
\end{abstract}

Results: A total of 66 women used DTG during pregnancy and the proportion on DTG increased each year: in 2015, 8\% (5/60) of women were on DTG, versus $22 \%(15 / 67)$ in $2016,42 \%(30 / 71)$ in 2017 , and 59\% $(16 / 27)$ in $2018(P<0.05)$. Among women who delivered $(n=57), 77.2 \%$ were undetectable at delivery. There were no drug resistance and no reported side effects during pregnancy. Infants had a mean APGAR score of 8 (SD 1.5) at $1 \mathrm{~min}$ and 9 (SD 0.8) at $5 \mathrm{~min} ; 31.6 \%$ were born prematurely and $15.8 \%$ were SGA, and 2 infants had a birth defect. No cases of HIV transmission occurred.

Conclusion: Our findings suggest that DTG can be an effective treatment during pregnancy. Infant outcomes (preterm deliveries and birth defects) need to be investigated in larger studies.

Copyright (C) 2018 The Author(s). Published by Wolters Kluwer Health, Inc.

AIDS 2018, 32:2017-2021

\begin{abstract}
${ }^{a}$ Drexel University College of Medicine, Philadelphia, Pennsylvania, ${ }^{b}$ Division of Infectious Diseases, Department of Medicine, Emory University School of Medicine, Atlanta, Georgia, ' Department of Community Health and Prevention, Drexel University Dornsife School of Public Health, Philadelphia, Pennsylvania, ${ }^{\mathrm{d}}$ Emory University School of Medicine, Atlanta, Georgia, ${ }^{\mathrm{e}}$ Division of Infectious Diseases and HIV Medicine, Department of Medicine, Drexel University College of Medicine, Philadelphia, Pennsylvania, ${ }^{f}$ AIDS Activities Coordinating Office, Philadelphia Department of Public Health, Philadelphia, Pennsylvania, ${ }^{\mathrm{g}}$ Department of Obstetrics \& Gynecology, Drexel University College of Medicine, Philadelphia, Pennsylvania, ${ }^{\mathrm{h}}$ Department of Pediatrics, Drexel University College of Medicine, Philadelphia, Pennsylvania, 'Division of Infectious Diseases, University of Alabama at Birmingham School of Medicine, Birmingham, Alabama, and 'Division of Maternal-Fetal Medicine, Department of Gynecology and Obstetrics, Emory University School of Medicine, Atlanta, Georgia, USA.

Correspondence to Florence Momplaisir, Division of Infectious Diseases and HIV Medicine, Department of Medicine, Drexel University College of Medicine, 1427 Vine Street, 4th Floor, Philadelphia, PA 19102, USA.
\end{abstract}

Tel: +1 631235 5911; fax: +1 215762 2531; e-mail: fmd38@drexel.edu

Received: 6 April 2018; revised: 29 May 2018; accepted: 12 June 2018. 


\section{Keywords: dolutegravir, HIV, infant health, maternal health, pregnant women}

\section{Introduction}

Dolutegravir (DTG), a second-generation HIV-1 integrase strand transfer inhibitor (INSTI), is a preferred regimen for adults living with HIV [1] and, until recently, was a second-line regimen for pregnant women living with HIV (WLWH) [2]. Emerging data from an ongoing $\mathrm{NIH}$-funded study in Botswana consisting of 5438 mother-infant pairs [3], revealed an increased risk of neural tube defects among infants of women who were on DTG at the time of conception. The study found a risk of neural tube defects of $0.9 \%$ (4/426) among infants exposed to DTG compared with $0.1 \%$ for infants exposed to other antiretrovirals (ART). On the basis of these results, the World Health Organization [4] and HIV treatment guidelines in Europe and the United States [57] now recommend against the use of DTG among women of childbearing age who intend to be pregnant or among pregnant women in their first trimester. Treatment guideline committees acknowledge that the neural tube defect findings are preliminary and advice may change as more data emerge.

Currently, studies of DTG use during pregnancy from resource-rich countries consist mostly of case studies showing DTG to be effective at achieving viral suppression and preventing perinatal HIV transmission, even in the setting of late presentation in prenatal care or resistant virus $[8,9]$. Pharmacokinetic studies show a reduced trough concentration in pregnancy but good placental and breastmilk penetration, which can be beneficial for protecting infants from perinatal HIV $[10,11]$. However, there remains a knowledge gap regarding viral suppression, development of resistance and neonatal outcomes from infants with in-utero exposure to DTG. Here, we describe the trends of DTG use of pregnant WLWH from two urban clinics, as well as maternal and neonatal outcomes (including any neural tube defects) at delivery.

\section{Methods}

\section{Patient population}

Two university-affiliated Ryan White-funded clinics with large prenatal populations participated in a multicenter retrospective cohort study: Drexel University College of Medicine, Philadelphia, Pennsylvania, USA and the Grady Infectious Diseases Program, Atlanta, Georgia, USA. This study was approved by the institutional review boards at Drexel and Emory Universities, and the Grady Research Oversight
Committee. A database search identified all female pregnant patients who received DTG during pregnancy, between 1 January 2015 and 28 May 2018. Treatmentnaive patients who initiated DTG in pregnancy, and treatment-experienced patients who were either already on DTG before pregnancy or were switched to DTG during pregnancy, were eligible for this study. We also queried the database to obtain the number of pregnant women who were on non-DTG-based regimen to obtain the percentage of DTG use over time. DTG could be given as a single tablet regimen with abacavir/lamivudine or in combination with other ART. Women with elective or spontaneous abortion, or with deliveries outside our institutions, were noted but not excluded. Demographic and clinical data were obtained on mother-infant pairs by chart review during pregnancy and up to delivery.

\section{Infant outcomes}

Infant outcomes included the detection of birth defects using a neonatal ultrasound or physician documentation at birth, preterm birth (gestational age of $<37$ weeks at delivery), small for gestational age (SGA, weight less than the 10th percentile for the gestational age), infant HIV status at birth by clinical documentation, and Appearance, Pulse, Grimace, Activity, Respiration (APGAR) scores at 1 and $5 \mathrm{~min}$.

\section{Maternal outcomes}

Maternal outcome variables were having an undetectable viral load of less than 20 copies/ml prior to delivery; documented resistance to either DTG or other ART; and tolerability to DTG, collected via physician documentation. For women who had already delivered, third trimester viral load closest to delivery was used. When delivery had not yet occurred, the last available viral load and the gestational age associated with date of collection were recorded. Demographic variables included maternal age at delivery, race/ethnicity, and maternal country of birth (US versus non-US born). Clinical variables included years living with HIV and HIV diagnosed before or during pregnancy. We reported the number of women on DTG prior to pregnancy who were also on DTG at the time of conception. For women previously on a non-DTG-based regimen who were started on a DTG-based regimen, we included the gestational age at the time of the switch. We reported the number of women, not previously on ART, who initiated a DTGbased regimen and the gestational age at DTG initiation.

\section{Analysis}

We described the proportion and characteristics of mother-infant pairs using DTG during the study period. For women who had not yet delivered by 22 May 2018, 


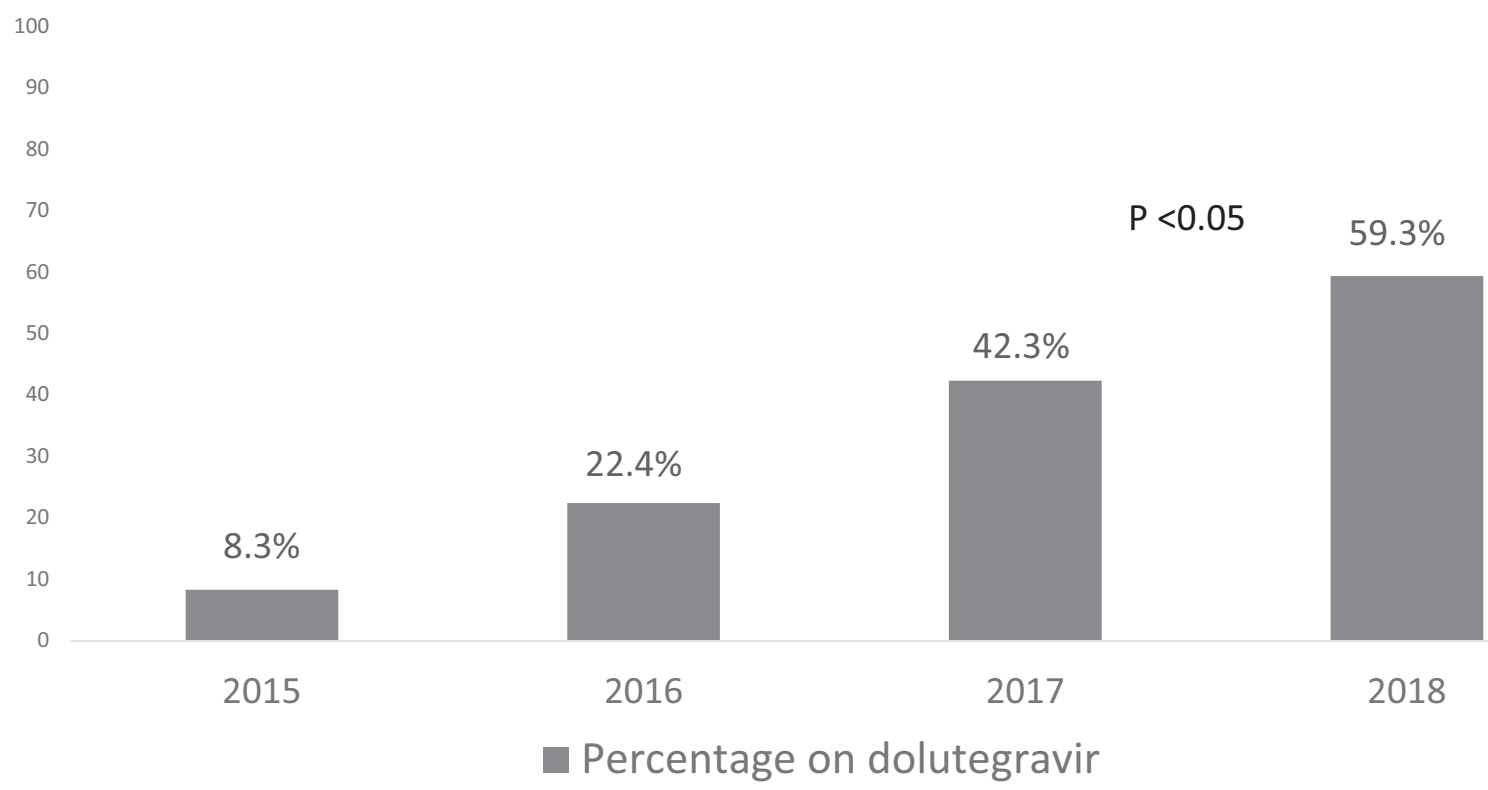

Fig. 1. The proportion of women with HIV using dolutegravir during pregnancy, 2015-2018. The total number of pregnancies was 60 in 2015, 67 in 2016, 71 in 2017 and 27 in 2018. 2018 includes January to May data only.

we collected study variables available up to the time of chart abstraction. Descriptive analyses included using mean and SD for continuous variables and proportion and associated SD for categorical variables. A trend analysis was performed to assess whether or not the proportion of women on DTG during pregnancy increased significantly over time.

\section{Results}

A total of 66 women used DTG during pregnancy and 57 had a live delivery during the study period. As demonstrated in Fig. 1, the proportion of women receiving DTG-based versus other regimens increased significantly by calendar year: $5 / 60(8.3 \%)$ women in $2015,15 / 67(22.4 \%)$ in $2016,30 / 71(42.3 \%)$ in 2017 , and $16 / 27(59.3 \%)$ from January to May $2018(P<0.05)$. Demographic and clinical variables are presented in Table 1. The majority of women were US born (80.3\%), black, non-Hispanic $(84.8 \%)$ with a mean age of 28 (SD 6) years and with preserved immunologic function $(84.8 \%$ had a $\mathrm{CD}^{+}>200$ cells/ $\left./ \mu \mathrm{l}\right)$. About $9 \%$ of women were diagnosed with HIV during pregnancy. Many women were on DTG before pregnancy and remained on DTG during pregnancy $(n=28,42.4 \%) ; 16(24.2 \%)$ initiated DTG and $22(33.3 \%)$ switched to DTG during pregnancy. The average gestational age for DTG initiation was 18.1 (SD 8.9) weeks and for regimen change was 21.1 (SD 10) weeks. The reasons for switching to DTG were to experience lower pill burden, lower side effects, or to achieve viral suppression before delivery. Once switched to DTG, women remained on it for the remainder of the pregnancy.

\section{Infant outcomes}

At birth, infants $(n=57)$ had a mean APGAR score of 8 (SD 1.5) at $1 \mathrm{~min}$ and 9 (SD 0.8) at $5 \mathrm{~min} ; 18$ (31.6\%) were preterm deliveries and $9(15.8 \%)$ were SGA. A total of two infants had a birth defect: one, from a twin pregnancy, developed nonimmune hydrops fetalis, and the second had a congenital heart abnormality (endocardial fibroelastosis versus ventricular septal defect). There were no cases of neural tube defects. All infants tested negative for HIV.

\section{Maternal outcomes}

Among women who delivered, 44 (77.2\%) had undetectable viral load at delivery. When considering the entire cohort, including those who had not yet delivered, $49(74.2 \%)$ had undetectable viral load. Two women in the cohort did not have delivery data because one moved out of the country while pregnant and the other had an elective termination of her pregnancy in the second trimester. A total of 16 women had pre-existing resistance to non-INSTIs; of these 16 women, six did not achieve viral suppression even after adding DTG to their regimen. However, no new resistance developed while on DTG, even among women with a history of prior ART resistance. There were no documented side effects from DTG during pregnancy.

\section{Discussion}

This study adds to the limited literature on DTG use among mother-infant pairs from resource-rich countries and provides some evidence for its safety and 'real world' effectiveness during pregnancy. We found no cases of 
Table 1. Characteristics of mother-infant pairs using dolutegravir during pregnancy from two large urban academic centers, January 2015 to May 2018.

\begin{tabular}{|c|c|c|c|}
\hline Maternal characteristics & Total, $n=66$ & Philadelphia, $n=35$ & Atlanta, $n=31$ \\
\hline Age, mean (SD) & $28.5(6)$ & $29(6)$ & $28(6)$ \\
\hline \multicolumn{4}{|l|}{ Race/ethnicity, $n(\%)$} \\
\hline Black, non-Hispanic & $56(84.8)$ & $27(77.1)$ & $29(93.5)$ \\
\hline White, non-Hispanic & $1(1.5)$ & $1(2.9)$ & $0(0.0)$ \\
\hline Hispanic & $8(12.1)$ & $6(17.1)$ & $2(6.5)$ \\
\hline Asian, Pacific Islander & $1(1.5)$ & $1(2.9)$ & $0(0.0)$ \\
\hline Non-US born & $13(19.7)$ & $6(17.1)$ & 7 (22.6) \\
\hline Years with HIV diagnosis, mean (SD) ${ }^{\mathrm{a}}$ & $9.9(7.7)$ & $9.8(8.4)$ & $10(7.0)$ \\
\hline $\mathrm{CD}^{+}{ }^{+}$greater than 200 at entry in prenatal care, $n(\%)$ & $56(84.8)$ & $31(88.6)$ & $25(80.6)$ \\
\hline HIV diagnosed before current pregnancy, $n(\%)$ & $60(90.9)$ & $30(85.7)$ & $30(96.8)$ \\
\hline DTG used before pregnancy, $n(\%)$ & $28(42.4)$ & $17(48.6)$ & $11(35.5)$ \\
\hline $\begin{array}{l}\text { Women not on ART at the beginning of pregnancy who } \\
\text { initiated DTG during pregnancy, } n(\%)\end{array}$ & $16(24.2)$ & $11(31.4)$ & $5(16.1)$ \\
\hline Gestational age at DTG initiation, mean weeks (SD) & $18.1(8.9)$ & $17.1(8.5)$ & $20.4(10.4)$ \\
\hline Change in regimen during pregnancy to include DTG, $n(\%)$ & $22(33.3)$ & $7(20.0)$ & $15(48.4)$ \\
\hline Gestational age of ART change, mean weeks (SD) & $21.1(10.0)$ & $17.6(8.7)$ & $23(10.5)$ \\
\hline Preexisting HIV resistance, $n(\%)$ & $16(24.2)$ & $7(20)$ & $9(29.0)$ \\
\hline Resistance developed while on DTG, $n(\%)$ & $0(0)$ & $0(0)$ & $0(0)$ \\
\hline${ }^{\mathrm{b}}$ Viral load less than 20 copies/ml using last available viral load, $n(\%)$ & $49(74.2)$ & $27(77.1)$ & $22(71.0)$ \\
\hline Viral load less than 20 copies among women who delivered, $n(\%)$ & $44 / 57(77.2)$ & $27 / 31(87.1)$ & $17 / 26(65.4)$ \\
\hline $\begin{array}{l}\text { Baseline viral load among women who either initiated or switched t } \\
\text { o DTG, copies } / \mathrm{ml}\end{array}$ & 14363 & 15192 & 13617 \\
\hline Average time to viral suppression, days (SD) & $48(23)$ & $40.4(18.6)$ & $59.7(27.3)$ \\
\hline Infant characteristics & $n=57$ & $n=31$ (includes two sets of twins) & $n=26$ \\
\hline APGAR score at $1 \mathrm{~min}$, mean (SD) & $8(1.5)$ & $8(1.4)$ & $8(2)$ \\
\hline APGAR score at 5 min, mean (SD) & $9(0.8)$ & $8.7(1)$ & $9(1)$ \\
\hline${ }^{\mathrm{c}}$ Preterm birth, $n(\%)$ & $18(31.6)$ & $9(29.0)$ & $9(34.6)$ \\
\hline${ }^{\mathrm{d}}$ Small for gestational age, $\mathrm{n}(\%)$ & $9(15.8)$ & $5(16.1)$ & $4(15.4)$ \\
\hline Birth defect, $n(\%)$ & $2(3.5)$ & $2(6.5)$ & $0(0)$ \\
\hline Infant HIV-positive status, n (\%) & $0(0)$ & $0(0)$ & $0(0)$ \\
\hline
\end{tabular}

Out of 66 pregnancies (35 in Philadelphia and 31 in Atlanta), so far, 57 have resulted in live deliveries. Two women in the cohort did not have delivery data because one moved out of the country while pregnant and the other had an elective termination of her pregnancy in the second trimester. ART: antiretroviral therapy; DTG: dolutegravir.

${ }^{a}$ There were a total of $12(18 \%)$ women who acquired HIV perinatally.

${ }^{\mathrm{b}}$ The HIV viral load used was the one closest to delivery if delivery occurred or the last available lab value at the time of chart abstraction if the woman had not yet delivered.

${ }^{\mathrm{c}}$ Preterm births: birth before 37 weeks.

${ }^{\mathrm{d}}$ Small for gestational age: weight less than 10 th percentile.

neural tube defects; however, the risk of neural tube defects needs to be assessed using provider reporting of pregnancies to the Antiretroviral Pregnancy Registry (APR) in the US and respective pregnancy registries used in other countries. We observed a significant increase in the proportion of women using DTG during pregnancy with rates of viral suppression at delivery that are similar to those previously reported from other US cohorts $[12,13]$. However, with the change of recent treatment guidelines, the number of women entering pregnancy on DTG or women who initiate DTG during pregnancy might significantly decrease.

Infants generally appeared healthy at birth based on APGAR scores. The percentage of birth defects we observed is similar to that reported in the APR [14] and in Europe [15]. Our cohort demonstrated a relatively high proportion of preterm births (31.6\%) and a similar proportion of SGA infants $(15.8 \%)$ compared to other cohorts of WLWH $[3,8]$; the proportion of preterm deliveries and SGA infants among pregnant WLWH receiving DTG-based therapy from other cohorts has ranged between $11-22 \%[14,16]$ and $17-25 \%[3,17]$, respectively, in resource-rich and resource-limited settings. Because of the short-term and long-term consequences of both preterm birth and SGA, future studies need to assess the safety profile ofDTG for infants, and explore potential mechanisms of adverse outcomes, such as the role of DTG in reducing estradiol in pregnancy [17].

This cohort had a viral suppression rate of $74.2 \%$. Studies using INSTIs late in pregnancy showed mixed results for viral suppression, but all resulted in reduced viral load at delivery $[9,18]$. A DTG pharmacokinetic study $(n=21)$ reported 100\% viral suppression [19]; however, patients with poor adherence are usually excluded from pharmacokinetic studies. In our study, a substantial number of women were diagnosed with HIV during pregnancy, had viral resistance, or switched regimens, all known predictors of viral nonsuppression at delivery $[2,20]$. Even with these barriers, most women achieved viral suppression, highlighting the potency of DTG during 
pregnancy. Furthermore, no resistance to DTG developed, which is consistent with findings in nonpregnant populations [21]. Although many women with preexisting resistance did not achieve suppression, this failure could potentially relate to issues of medication access or adherence.

Although we offered a larger cohort than most previous studies, limitations include the retrospective nature of data collection. Our study adds to the current literature by providing evidence from a real-word cohort that DTG is effective at suppressing plasma viral load. However, specific effects on infants should be explored further.

\section{Acknowledgements}

\section{Conflicts of interest}

There are no conflicts of interest.

\section{References}

1. Panel on Antiretroviral Guidelines for Adults and Adolescents. Guidelines for the Use of Antiretroviral Agents in Adults and Adolescents Living with HIV. Department of Health and Human Services. Available at: http://www.aidsinfo.nih.gov/ContentFiles/AdultandAdolescentGL.pdf. (Accessed 29 May 2018)

2. Panel on Treatment of Pregnant Women with HIV Infection and Prevention of Perinatal Transmission. Recommendations for Use of Antiretroviral Drugs in Transmission in the United States. Available at: http://aidsinfo.nih.gov/contentfiles/lvguidelines/ PerinatalGL.pdf. (Accessed 29 May 2018)

3. Zash R, Jacobson DL, Diseko M, Mayondi G, Mmalane M, Essex $M$, et al. Comparative safety of antiretroviral treatment regimens in pregnancy. IAMA Pediatr 2017; 171:e172222-e1172222.

4. WHO. Statement on DTG-Geneva 18 May 2018. Available at: http://www.who.int/medicines/publications/drugalerts/Statement on_DTG_18May_2018final.pdf?ua=1. (Accessed 29 May 2018)

5. BHIVA. Current Guidelines. Available at http://www.bhiva.org/ guidelines.aspx. Accessed on May 29, 2018.

6. European Medicines Agency. New study suggests risk of birth defects in babies born to women on HIV medicine dolutegravir. Available at: http://www.ema.europa.eu/ema/index.jsp?curl=pages/news_and_events/news/2018/05/news_detail_002956.jsp\&mid=WC0b01ac058004d5c1. (Accessed 29 May 2018)

7. HHS Antiretroviral Guidelines Panels. Statement on Potential Safety Signal in Infants Born to Women Taking Dolutegravir from the HHS Antiretroviral Guideline Panels. Available at: https://aidsinfo.nih.gov/news/2094/statement-on-potentialsafety-signal-in-infants-born-to-women-taking-dolutegravir. (Accessed on 29 May 2018)
8. Pinnetti $C$, Tintoni $M$, Ammassari A, Tamburrini E, Bernardi S, Liuzzi $G$, et al. Successful prevention of HIV mother-to-child transmission with dolutegravir-based combination antiretroviral therapy in a vertically infected pregnant woman with multiclass highly drug-resistant HIV-1. AIDS 2015; 29:2534-2537.

9. Rahangdale L, Cates J, Potter J, Badell ML, Seidman D, Miller ES, et al. Integrase inhibitors in late pregnancy and rapid HIV viral load reduction. American journal of obstetrics and gynecology 2016; 214:385e381-e385, e387.

10. Rimawi BH, Johnson E, Rajakumar A, Tao S, Jiang Y, Gillespie S, et al. Pharmacokinetics and placental transfer of elvitegravir, dolutegravir, and other antiretrovirals during pregnancy. Antimicrob Agents Chemother 2017; 61:pii: e02213-16.

11. Mulligan N, Best BM, Wang J, Capparelli EV, Stek A, Barr E, et al. Dolutegravir pharmacokinetics in pregnant and postpartum women living with HIV. AIDS 2018; 32:729-737.

12. Swain C-A, Smith LC, Nash D, Pulver WP, Lazariu V, Anderson BJ, et al. Postpartum loss to HIV care and HIV viral suppression among previously diagnosed HIV-infected women with a live birth in New York State. PloS One 2016; 11: e0160775.

13. Katz IT, Leister E, Kacanek D, Hughes MD, Bardeguez A Livingston $E$, et al. Factors associated with lack of viral suppression at delivery among highly active antiretroviral therapynaive women with HIV: a cohort study. Ann Intern Med 2015; 162:90-99.

14. Vannappagari $\mathrm{V}$, Albano J, Ragone A, Scheuerle A, Tilson $\mathrm{H}$, Cook T, et al. Dolutegravir use during pregnancy and birth outcomes: data from the Antiretroviral Pregnancy Registry (APR). In: 9th International AIDS Society Conference. Paris, France; 2017.

15. Thorne C, Favarato G, Peters H, Soriano-Arandes T, Floridia M, Colbers A, et al. Pregnancy and neonatal outcomes following prenatal exposure to dolutegravir. In: International AIDS Society Conference. Paris, France; 2017.

16. Katz IT, Leister E, Kacanek D, Hughes MD, Bardeguez A, Livingston $E$, et al. Factors associated with lack of viral suppression at delivery among highly active antiretroviral therapy-naive women with HIV: a cohort study. Ann Intern Med 2015; 162:90-99.

17. Powis KJ, Legbedze, J, Mmasa KN, Kgole SW, Kala S, Bhattacharya A, et al. Estradiol levels in HIV infected pregnant women on dolutegravir-based ART in Botswana. In: 8th International Workshop on HIV and Women. Boston, Massachusetts, 2018.

18. Mounce ML, Pontiggia L, Adams IL. A single-center retrospective cohort analysis of maternal and infant outcomes in HIVinfected mothers treated with integrase inhibitors during pregnancy. Infect Dis Ther 2017; 6:531-544.

19. Mulligan N, Best B, Capparelli E, Stek A, Barr E, Smith E, et al. Dolutegravir pharmacokinetics in HIV-infected pregnant and postparum women. In: Conference on Retroviruses and Opportunistic Infections. Boston, Massachusetts, 2016.

20. Momplaisir FM, Brady KA, Fekete T, Thompson DR, Diez Roux $A$, Yehia BR. Time of HIV diagnosis and engagement in prenatal care impact virologic outcomes of pregnant women with HIV. PLoS One 2015; 10:e0132262.

21. Clotet B, Feinberg J, Van Lunzen J, Khuong-Josses M-A, Antinori A, Dumitru I, et al. Once-daily dolutegravir versus darunavir plus ritonavir in antiretroviral-naive adults with HIV-1 infection (FLAMINGO): 48 week results from the randomised openlabel phase 3b study. Lancet 2014; 383:2222-2231. 